\title{
Desórdenes temporomandibulares durante la pandemia por el COVID-19

\author{
Leopoldo Meneses-Rivadeneira'
}

\section{Resumen}

Después de 2 años de haberse identificado en Wuhan - China la infección respiratoria causada por el SARS-CoV-2, que ha provocado la última pandemia global, seguimos viviendo la incertidumbre de lo que se sabe, de lo que aún no se sabe y observando lo que nos va dejando la pandemia. Dentro de los múltiples problemas de la salud que se han ocasionado por la pandemia, los problemas estomatológicos no solo se han incrementado por la falta de atención, si no que han cambiado algunos datos epidemiológicos que los caracterizaba. Es así que los desórdenes temporomandibulares (DTM) casi se han duplicado en número en adolescentes y adultos jóvenes, lo cual se asocia a las múltiples reacciones psicológicas adversas que han generado miedo excesivo, ansiedad y depresión por la pandemia del COVID-19. Es propósito de esta publicación presentar una revisión del contexto actual de los desórdenes temporomandibulares.

Palabras claves: COVID-19, atención estomatológica, desórdenes temporomandibulares.

Abstract

After two years of having identified in Wuhan - China the respiratory infection caused by SARS-CoV-2 that has caused the last global pandemic, we continue to experience the uncertainty of what is known, what is not yet known and observing what that the pandemic is leaving us. Among the many health problems that have been caused by the pandemic, stomatological problems have not only increased due to lack of care, but also some epidemiological data that characterized them have changed. Thus, temporomandibular disorders (TMD) have almost doubled in number in adolescents and young adults, which is associated with the multiple adverse psychological reactions that have generated excessive fear, anxiety and depression due to the COVID-19 pandemic. The purpose of this publication is to present a review of the current context of temporomandibular disorders.

Keywords: COVID-19, dental care, temporomandibular disorders.

Introducción

A fines del año 2019 en Wuhan - China, se identificó un nuevo coronavirus que se denominó SARS-CoV-2 y que dio partida a la infección respiratoria más agresiva y mortal, responsable de la pandemia por el COVID -19. Actualmente, la pandemia ya cobró más de 4 millones de vida en todo el mundo, existe la aparición de nuevas cepas y nuevos rebrotes en diversos países que no pueden ser revertidos por la mayoría de sistemas de salud pese a la vacunación, que por cierto no es equitativa a nivel global $^{(1)}$.
Dentro de los múltiples problemas de la salud que se han ocasionado por la pandemia, están problemas estomatológicos, los cuales no solo se han incrementado por la falta de atención, si no que han cambiado algunos datos epidemiológicos que los caracterizaba. Es así que hoy en día casi se han duplicado el número de DTM en adolescentes y adultos jóvenes, lo cual se asocia a las múltiples reacciones psicológicas adversas que han generado miedo excesivo, ansiedad y depresión por la pandemia del COVID $-19^{(2)}$.

${ }^{1}$ Cirujano - Dentista, Profesor Asociado Universidad Peruana Cayetano Heredia. Past Presidente de la Asociación Peruana de Cirugía Bucal y Maxilofacial. Past Presidente de la Asociación Latinoamericana de Cirugía y Traumatología Bucal y Maxilofacial. ORCID: https://orcid.org/0000-0003-0905-506X. 
La etiología de los DTM es multifactorial, incluye la acción combinada de factores ambientales, biológicos, psicológicos, biomecánicos y neuromusculares. Los síntomas son generalmente dolor de mandíbula, dolor de oído, dolor de dientes (no odontogénico), dolor en las articulaciones, dolor de cabeza, y limitación funcional mandibular. El 50\% de la población presenta algún nivel de DTM, pero solo del 3,6\% al $7 \%$ requieren tratamiento ${ }^{(3,4-6,)}$.

La importancia de los factores psicosociales en el desarrollo y curso de los DTM se han descrito en muchos estudios previos, así como la relación de los DTM dolorosos con la depresión y ansiedad debido a que todos estos eventos conllevan a una sobrecarga funcional de la oclusión asociada al estrés. Asimismo, se ha encontrado asociación entre el bruxismo del despierto con los trastornos de ansiedad y estrés más que en el bruxismo del sueño de tal forma que los pacientes con altos niveles de estrés tienen casi 6 veces más probabilidades de desarrollar el bruxismo del despierto ${ }^{(7-9)}$.

Medeiros y colaboradores en Brasilia-Brasil, realizaron un estudio de prevalencia de síntomas de trastornos temporomandibulares, conductas bucales, ansiedad y depresión en estudiantes de odontología durante el período de aislamiento social por COVID - 19. De un total de 113 estudiantes 87 fueron mujeres $(77 \%)$ y 26 hombres $(23 \%)$, con una edad media de $21,46 \pm 2,37$ años, encontraron que $56(49,6 \%)$ estudiantes tuvieron algún síntoma de ansiedad y 44 (38.9\%) algún síntoma de depresión; sin embargo, solo 31 estudiantes (27.4\%) presentaron síntomas de DTM doloroso, por lo que no encontraron asociación entre DTM sintomático con ansiedad y depresión, pero sí un incremento de casos en este grupo etario ${ }^{(3)}$.

Saccomanno y colaboradores en Italia, realizaron un estudio para evaluar si el aislamiento social del coronavirus como un factor importante de estrés en la vida afectaba los síntomas de los DTM, encontrando que de 182 sujetos (52 hombres y 130 mujeres), con una edad promedio de 45 años, 74 $(40.6 \%)$ sujetos que informaron síntomas de DTM, de los cuales $45(60,8 \%)$ informaron un inicio reciente dolor facial durante los meses de aislamiento social y 38 (51,4\%) informó que estos síntomas empeoraron en el último mes; de éstos últimos 35 (94,7\%) informaron que el agravamiento del dolor se debió al encierro estresante y sus consecuencias ${ }^{(5)}$.

Vrbanovié y colaboradores, Zagred-Croacia, evaluaron los efectos de la pandemia del COVID-19 y los terremotos de Zagreb como factores estresantes en pacientes con trastornos temporomandibulares, encontrando que de 53 pacientes tratados previamente por DTM que viven en la zona de los sismos, con una edad promedio de $33.6 \pm 11.4$, catorce $(26,4 \%)$ informaron la aparición de nuevos síntomas después del ascenso del COVID-19, y 22 (41,5\%) informaron la aparición de nuevos síntomas después de terremotos ${ }^{(10)}$.

Asquini y colaboradores en Milan-Italia, realizaron el primer estudio prospectivo que describe cómo afecta la angustia relacionada al COVID-19 a personas con DTM durante el encierro en relación con su salud general, conductas orales, características psicosociales, discapacidad e intensidad del dolo; encontrando que de 45 personas con DTM crónico, 10 participantes presentaron un trastorno de la articulación temporomandibular, 12 a trastornos de los músculos masticatorios y 23 un trastorno mixto. El conocimiento obtenido en este estudio contribuye a una mayor comprensión del papel del estrés psicológico como un posible amplificador de la escala de estrés por el COVID-19, ansiedad, depresión, dolor crónico y discapacidad relacionada con el dolor en personas con $\mathrm{DMT}^{(11)}$.

\section{Etiología de los desórdenes temporomandibulares}

El término DMT no es un diagnóstico, es un término amplio que incluye una serie de enfermedades como el dolor en los músculos masticatorios y articulaciones temporomandibular, dolor de cabeza, alteraciones en la mandíbula movimientos y sonidos en las articulaciones al abrir y cerrar la boca. Las causas de estas enfermedades y/o síntomas son numerosos e incluyen traumatismos, sistémicos, iatrogénicos, trastornos oclusales y de salud mental. Cabe resaltar que para que esto ocurra se debe superar el alto potencial que el sistema neuromuscular tiene para adaptarse a la función masticatoria, solo estas capacidades compensatorias se sobrecargan y se produce una disfunción que da lugar a síntomas clínicos como dolor, chasquidos intensos o movilidad limitada de la mandíbula ${ }^{(12)}$ (Tabla 1).

\section{Clasificación de los desórdenes temporomandibulares}

Para fines prácticos de recordar los tipos de DTM, se pueden mencionar que son tres los trastornos más comunes: dolor y disfunción miofascial, trastornos internos y osteoartrosis. El dolor y disfunción miofascial es el más prevalente, siendo un trastorno muscular resultante de hábitos orales parafuncionales como el apretamiento o bruxismo, que a su vez se pueden relacionar con trastornos psicógenos como dolor de cabeza, fibromialgia, dolor de espalda crónico e irritable síndrome del intestino con estrés, ansiedad y depresión. El trastorno interno es utilizado para describir un trastorno 


\begin{tabular}{|c|c|}
\hline \multicolumn{2}{|r|}{ Teorías sobre el origen de los DTM } \\
\hline Nombre de la teoría & Fundamentos \\
\hline $\begin{array}{l}\text { Desplazamiento mecánico } \\
\text { (por Costen) }\end{array}$ & $\begin{array}{l}\text { La falta de apoyo en los dientes laterales o los contactos prematuros oclusales funcionales conducen a un } \\
\text { posicionamiento excéntrico directo del cóndilo en la fosa glenoidea; esto conduce a dolor, síntomas de oído, } \\
\text { actividad muscular adversa y DMT. }\end{array}$ \\
\hline $\begin{array}{l}\text { Teoría del trauma } \\
\text { (por Zack y Speck) }\end{array}$ & $\begin{array}{l}\text { El factor principal de DMT es el micro / macro-trauma; El trauma puede causar alteración estructural a los } \\
\text { músculos o directamente a las estructuras articulares. }\end{array}$ \\
\hline $\begin{array}{l}\text { El trastorno biomédico } \\
\quad \text { (por Reade) }\end{array}$ & $\begin{array}{l}\text { Es iniciado por un trauma; factores específicos (maloclusión, parafunciones, actividades ocupacionales) } \\
\text { provocan la progresión de síntomas. }\end{array}$ \\
\hline $\begin{array}{c}\text { Osteoartric } \\
\text { (por Stegenga) }\end{array}$ & $\begin{array}{l}\text { La osteoartrosis es una causa principal de DMT; Los síntomas musculares y las enfermedades sistémicas } \\
\text { son secundarios a la patología de la ATM. }\end{array}$ \\
\hline $\begin{array}{l}\text { Múscular } \\
\text { (por Travell y Rinzler) }\end{array}$ & $\begin{array}{l}\text { Los músculos masticatorios son el factor etiológico principal de DMT; La mialgia (causada por mioespasmo } \\
\text { crónico) es secundaria a parafunciones y puede referir el dolor a la ATM. }\end{array}$ \\
\hline $\begin{array}{l}\text { Neuromuscular } \\
\text { (por Ramfjord) }\end{array}$ & $\begin{array}{l}\text { Los problemas oclusales causan DMT, la pérdida del equilibrio oclusal conduce a la descoordinación de los } \\
\text { músculos y los espasmos. }\end{array}$ \\
\hline $\begin{array}{l}\text { Psicofisiológico } \\
\text { (por Schwartz y Laskin) }\end{array}$ & $\begin{array}{l}\text { DMT ocurre fuera de los factores físicos; Los factores psicosociales juegan un papel crucial en la } \\
\text { patogénesis de los DMT: el principal factor de hipertensión y sobrecontracción del músculo se debe a las } \\
\text { parafunciones realizadas para aliviar el estrés. }\end{array}$ \\
\hline $\begin{array}{l}\text { Teoría psicosocial } \\
\text { (por Dworkin) }\end{array}$ & $\begin{array}{l}\text { Los trastornos emocionales inducen hiperactividad de los músculos y conducen a hábitos parafuncionales y } \\
\text { anomalías oclusales; la contractividad muscular se acentúa con el apretamiento de los dientes y la } \\
\text { repetibilidad conduce al dolor, }\end{array}$ \\
\hline
\end{tabular}

Adaptado de Bhat S. Etiology of temporomandibular disorders: the journey so far.Int Dent SA. 2010, 12:88-92. Citado en ${ }^{12}$

temporomandibular donde hay una posición anormal del disco articular resultando en interferencia mecánica (clic articular) y la restricción del rango normal de actividad mandibular (apertura bucal limitada) o incluso hipermovilidad que puede resultar en la dislocación de la mandíbula. La osteoartrosis es un trastorno degenerativo localizado que afecta principalmente al cartílago articular del cóndilo mandibular de la articulación temporomandibular y se ve a menudo en personas mayores, pero puede presentarse con poca frecuencia en jóvenes ${ }^{(13)}$ (Tabla 2).

\section{Diagnóstico por imágenes de los desórdenes temporomandibulares}

Las imágenes pueden ayudar en el diagnóstico de los DTM cuando la historia y los hallazgos del examen físico son equívocos. El estudio inicial debe ser una radiografía panorámica en las que se pueden apreciar fracturas agudas, dislocaciones y enfermedad articular degenerativa grave. La tomografía computarizada es superior a la radiografía simple para la evaluación de la morfología sutil ósea.
La resonancia magnética es la modalidad óptima de evaluación integral conjunta en pacientes con signos y síntomas de los DTM demostrando una correlación de cambios de la morfología articular y sintomatología patológica entre $78 \%$ y $95 \%$. Si embargo, existen entre un $20 \%$ a $34 \%$ de falsos positivos en pacientes asintomáticos. Las imágenes de resonancia magnética se deben reservar en los casos de sintomatología persistente, en aquellos casos con terapia conservadora ineficaz, o en aquellos con sospecha de trastorno $\operatorname{articularinterno}^{(14)}$.

\section{Tratamiento de los desórdenes temporomandibulares}

Los principales objetivos del tratamiento de los DTM son reducir o eliminar el dolor y/o ruidos articulares para restaurar la función mandibular normal. Esto se logra mejor cuando otros factores contribuyentes tales como estrés, depresión y hábitos orales parafuncionales, como el bruxismo, se abordan e incorporan en la estrategia general de tratamiento. Es esencial que el clínico establezca si el problema es físico o 


\begin{tabular}{|c|c|}
\hline \multicolumn{2}{|c|}{ Tabla 2} \\
\hline \multicolumn{2}{|c|}{ Principales desórdenes temporomandibulares } \\
\hline \multicolumn{2}{|c|}{ 1. Dolor y disfunción miofascial } \\
\hline a. & Miositis \\
\hline b. & Fibromialgia \\
\hline c. & Dolor neuropático \\
\hline d. & Síndrome de dolor crónico \\
\hline \multicolumn{2}{|c|}{ 2. Trastorno funcional de la ATM } \\
\hline a. & Trastorno interno - desplazamiento del disco \\
\hline b. & Trastornos de hipermovilidad: luxación \\
\hline c. & Trastornos de la hipomovilidad: anquilosis, postraumática \\
\hline \multicolumn{2}{|c|}{ 3. Enfermedad articular degenerativa / inflamatoria de la ATM } \\
\hline a. & Osteoartrosis / artritis \\
\hline b. & Artritis reumatoide \\
\hline c. & Artritis psoriásica \\
\hline d. & Artritis juvenil \\
\hline
\end{tabular}

Adaptado de13

\section{Tabla 3}

\section{Estrategias de manejo de los DTM}

\section{Explicación y tranquilidad}

a. DMT no pone en peligro la vida

b. DMT no es un cáncer

c. DMT puede convertirse en una condición crónica

d. DMT puede ser manejada

2. Educación y autocuidado
a. Dieta blanda
b. Descanso de la mandíbula (especialmente durante consultas dentales prolongadas)
c. Evite movimientos extremos de la mandibula (es decir, bostezos)
d. Calor tópico
e. Proteja la cara y las mandíbulas del clima frío
f. Evite el estrés y la ansiedad

\section{Medicamentos}
a. Antiinflamatorios
b. Ansiolíticos
c. Relajantes musculares
d. Antidepresivos

\section{Fisioterapia de la mandíbula}
a. Masaje y estiramiento
b. Punción seca
c. TENS: estimulación nerviosa eléctrica transcutánea
d. Terapia de ultrasonido pulsado 


\section{Tabla 3 (continuación)}

5. Terapia con aparato oclusal

6. Terapia conductual
a. Asesoramiento sobre estilo de vida
b. Terapia de relajación
c. Hipnosis
d. Biofeedback

7. Psicoterapia

8. Otro
a. Acupuntura
b. Inyecciones de Botox
c. Manipulación quiropráctica

9. Cirugía de ATM

9.1. Procedimientos cerrados

a. Artrocentesis de la ATM

b. Artroscopia de ATM

9.2. Procedimientos abiertos

a. Artrotomía / artroplastia de ATM

b. Reemplazos de articulaciones de la ATM

Adaptado de13

psicógeno, ya que esto determinará el tratamiento. La mayoría de los trastornos psicógenos se encuentran en el grupo de disfunción y dolor miofascial de los trastornos temporomandibulares que necesitarán psicofármacos y psicoterapia ${ }^{(7)}$ (Tabla 3).

\section{Conclusiones}

Sin duda la pandemia por el COVID-19 nos va dejando secuelas propias de la enfermedad y otras que se han incrementado por falta de atención durante los largos períodos de aislamiento social que se tiene que mantener para evitar el contagio y diseminación. Es estomatología los DTM no solo se incrementaron en la población más prevalente, sino que se está observando un incremento significativo en la población adolescente y adulta joven que hasta antes de la pandemia no se afectaba con frecuencia. Entendiendo que los DTM pueden llegar a ser muy degenerativos con el tiempo, los odontólogos deben estar muy atentos a diagnosticar y establecer el tratamiento multidisciplinario apropiado lo más oportunamente posible. La derivación a los odontólogos especialistas en rehabilitación oral y cirugía bucal y maxilofacial se debe considerar para que los diversos tratamientos de los DTM se pueden adaptar específicamente a cada uno de los casos diagnosticados.

\section{Referencias bibliográficas}

\section{Huang C, Wang Y, Li X, Ren L, Zhao J, Hu Y, et al. Clinical} features of patients infected with 2019 novel coronavirus in Wuhan, China.Lancet. 2020;395(10223):497-506. Erratum in: Lancet. 2020 Jan 30. doi:10.1016/S0140-6736(20)30183-5.

2. Almeida-Leite CM, Stuginski-Barbosa J, Conti PCR. How psychosocial and economic impacts of COVID-19 pandemic can interfere on bruxism and temporomandibular disorders? J Appl Oral Sci. 2020;28:e20200263. doi: 10.1590/1678-77572020-0263.

3. Medeiros RA, Vieira DL, Silva EVFD, Rezende LVML, Santos RWD, Tabata LF. Prevalence of symptoms of temporomandibular disorders, oral behaviors, anxiety, and depression in Dentistry students during the period of social isolation due to COVID-19. J Appl Oral Sci. 2020 ;28:e20200445. doi: 10.1590/1678-7757-2020-0445.

4. Bertoli FM, Bruzamolin CD, Kranz GO, Losso EM, Brancher JA, Souza JF. Anxiety and malocclusion are associated with temporomandibular disorders in adolescents diagnosed by RDC/TMD: a cross-sectional study. J Oral Rehabil. 2018;45(10):747-755. doi:10.1111/joor.12684

5.Saccomanno S, Bernabei M, Scoppa F, Pirino A, Mastrapasqua R, Visco MA. Coronavirus Lockdown as a 
Major Life Stressor: Does It Affect TMD Symptoms? Int J Environ Res Public Health. 2020;17(23):8907. doi:10.3390/ijerph17238907.

6. Callahan, CD. Stress, coping, and personality hardiness in patients with temporomandibular disorders. Rehabil Psychol. 2000;45:38-48. doi:org/10.1037/0090-5550.45.1.38.

7. Manfredini D, Lombardo L, Siciliani G. Temporomandibular disorders and dental occlusion. A systematic review of association studies: end of an era? J Oral Rehabil. 2017;44(11):908-23. doi: 10.1111/joor.12531.

8. Boscato N, Almeida RC, Koller CD, Presta AA, Goettems ML. Influence of anxiety on temporomandibular disorders: an epidemiological survey with elders and adults in Southern Brazil. J Oral Rehabil. 2013;40(9):643-649. doi:10.1111/joor. 12076.

9.Smardz J, Martynowicz H, Wojakowska A, MichalekZrabkowska M, Mazur G, Wieckiewicz M. Correlation between sleep bruxism, stress, and depression-A polysomnographic study. J Clin Med. 2019;8(9):1344. doi: $10.3390 /$ jcm8091344

10.Vrbanovié E, Alajbeg IZ, Alajbeg I. COVID-19 pandemic and Zagreb earthquakes as stressors in patients with temporomandibular disorders. Oral Dis. 2021;27 Suppl 3:688693. doi:10.1111/odi.13488.

11. Asquini G, Bianchi AE, Borromeo G, Locatelli M, Falla D. The impact of Covid-19-related distress on general health, oral behaviour, psychosocial features, disability and pain intensity in a cohort of Italian patients with temporomandibular disorders. PLoS One. 2021;16(2):e0245999. doi:10.1371/ journal.pone.0245999.

12.Wieckiewicz M, Boening K, Wiland P, Shiau YY, Paradowska-Stolarz A. Reported concepts for the treatment modalities and pain management of temporomandibular disorders. J Headache Pain. 2015;16:106. doi:10.1186/s10194015-0586-5.

13. Dimitroulis G. Management of temporomandibular joint disorders: A surgeon's perspective. Aust Dent J. 2018;63 Suppl 1:S79-S90. doi:10.1111/adj.12593.

14. Gauer RL, Semidey MJ. Diagnosis and treatment of temporomandibular disorders. Am Fam Physician. 2015 ;91(6):378-86.

Contribución de autoría: Leopoldo Meneses-Rivadeneira ha sido el autor de esta publicación, contribuyendo a su concepción, organización de la presentación científica, búsqueda electrónica, revisión inicial, redacción, revisión y aprobación final.

Conflicto de interés: El autor no tiene conflicto de interés con la publicación de este trabajo. Financiamiento: Autofinanciado.

Citar como: Meneses-Rivadeneira L. Desórdenes temporomandibulares durante la pandemia por el COVID-19. Diagnóstico (Lima). 2022;61(1):27-32.

DOI: https://doi.org/10.33734/diagnostico.v61i1.346

Correspondencia: Leopoldo Meneses Rivadeneira.

Correo electrónico:lvmeneses@gmail.com

Teléfono: 999004510

\section{Central Telefónica}
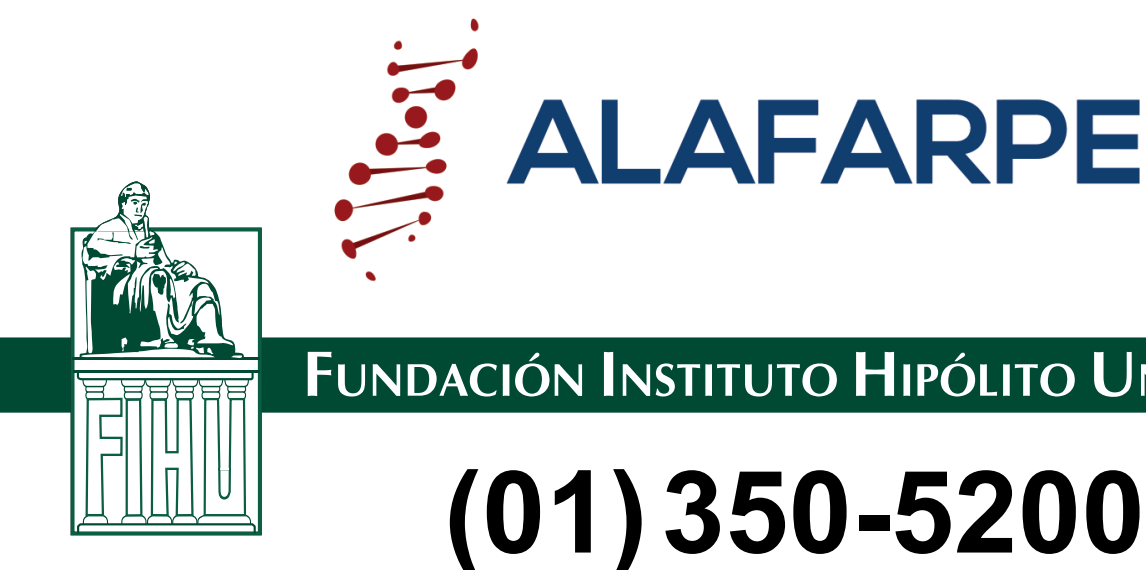

FundaCión INSTITUTO HIPÓLITO UNANUE

\section{(01) $350-5200$}

